\title{
IRE1a/XBP1-mediated branch of the unfolded protein response regulates osteoclastogenesis
}

\author{
Takahide Tohmonda, ${ }^{1,2}$ Masaki Yoda, ${ }^{1}$ Takao Iwawaki, ${ }^{3}$ Morio Matsumoto, ${ }^{4}$ Masaya Nakamura, ${ }^{4}$ Katsuhiko Mikoshiba, ${ }^{2}$ \\ Yoshiaki Toyama, ${ }^{4}$ and Keisuke Horiuchi ${ }^{1,4}$ \\ 'Department of Anti-Aging Orthopedic Research, Keio University School of Medicine, Tokyo, Japan. ²aboratory for Developmental Neurobiology, RIKEN Brain Science Institute, Wako, Saitama, Japan. \\ ${ }^{3}$ Iwawaki Lab, Advanced Scientific Research Leaders Development Unit, Gunma University, Maebashi, Gunma, Japan. ${ }^{4}$ Department of Orthopedic Surgery, Keio University School of Medicine, Tokyo, Japan.
}

\begin{abstract}
The unfolded protein response (UPR) is a cellular adaptive mechanism that is activated in response to the accumulation of unfolded proteins in the endoplasmic reticulum. The inositol-requiring protein-1 $\alpha / X$-box-binding protein-mediated (IRE1 $\alpha$ / XBP1-mediated) branch of the UPR is highly conserved and has also been shown to regulate various cell-fate decisions. Herein, we have demonstrated a crucial role for the IRE $\alpha$ /XBP1-mediated arm of the UPR in osteoclast differentiation. Using murine models, we found that the conditional abrogation of IRE1 $\alpha$ in bone marrow cells increases bone mass as the result of defective osteoclastic bone resorption. In osteoclast precursors, IRE1 $\alpha$ was transiently activated during osteoclastogenesis, and suppression of the IRE1 $\alpha /$ XBP1 pathway in these cells substantially inhibited the formation of multinucleated osteoclasts in vitro. We determined that XBP1 directly binds the promoter and induces transcription of the gene encoding the master regulator of osteoclastogenesis nuclear factor of activated T cells cytoplasmic 1 (NFATc1). Moreover, activation of IRE1 $\alpha$ was partially dependent on $\mathrm{Ca}^{2+}$ oscillation mediated by inositol 1,4,5-trisphosphate receptors 2 and 3 (ITPR2 and ITPR3) in the endoplasmic reticulum, as pharmacological inhibition or deletion of these receptors markedly decreased Xbp1 mRNA processing. The present study thus reveals an intracellular pathway that integrates the UPR and osteoclast differentiation through activation of the IRE1 $\alpha /$ XBP1 pathway.
\end{abstract}

\section{Introduction}

Various endogenous and exogenous factors, including hypoxia, starvation, oxidative stress, and protein synthesis overload, lead to an accumulation of unfolded proteins in the ER lumen, a condition referred to as ER stress. To comply with the burden in the ER and to normalize its functions, cells are equipped with machinery called the unfolded protein response (UPR). In mammalian cells, there are 3 major ER stress sensors that regulate the homeostasis of ER functions and ER stress-induced cell death (1-6): activating transcription factor 6 (ATF6), pancreatic ER kinase (PERK), and serine/threonine-protein kinase/endoribonuclease inositol-requiring protein-1 $\alpha$ (IRE1 $\alpha$ ). IRE1 $\alpha$ is a transmembrane protein that is localized in the ER and harbors both a kinase domain and an endonuclease domain in its cytoplasmic tail. Upon stimulation by ER stress, IRE1 $\alpha$ processes X-boxbinding protein $(X b p 1)$ transcripts, resulting in a splice variant of $X b p 1$ mRNA (Xbp1s) (7). Xbp1s mRNA encodes the transcription factor $\mathrm{XBP} 1 \mathrm{~s}$, which induces an array of genes involved in the recovery of ER functions $(8,9)$. The IRE1 $\alpha / \mathrm{XBP} 1$ pathway is the most evolutionarily conserved branch of the UPR (4); however, past studies revealed that this pathway also possesses functions that are not directly related to the UPR, including the regulation of innate immunity, energy metabolism, and cell differentiation $(8,10-15)$. These observations suggest that the IRE1 $\alpha / \mathrm{XBP} 1$ pathway is a critical component that integrates the UPR with the regulation of various cell-fate decisions.

Conflict of interest: The authors have declared that no conflict of interest exists. Submitted: April 28, 2014; Accepted: June 9, 2015.

Reference information: J Clin Invest. 2015;125(8):3269-3279. doi:10.1172/JCI76765.
Osteoclasts are derived from the monocyte/macrophage cell lineage and are essential for bone resorption and remodeling (16-18). Osteoclastic activity is intricately regulated by various hormones and cytokines to balance bone resorption with the formation of mineralized bone by osteoblasts. The coupling of bone resorption and bone formation is critical for the maintenance and homeostasis of the skeletal system, and its deterioration often results in overt osteolytic activity, leading to pathological conditions such as osteoporosis, bone metastasis, and arthritic bone destruction (19-21). Therefore, understanding how the differentiation and activity of osteoclasts are regulated is clinically relevant and critical. At a molecular level, the differentiation of osteoclasts is triggered by the binding of RANK, which is expressed on osteoclast precursor cells, with RANKL, a membrane-bound protein that is expressed on osteoblasts and osteocytes. Upon ligand binding, RANK activates various signaling pathways and induces intracellular $\mathrm{Ca}^{2+}$ oscillation, ultimately leading to the activation of the transcription factors essential for osteoclastogenesis, including c-Fos and the nuclear factor of activated $\mathrm{T}$ cells cytoplasmic 1 (NFATc1) $(22,23)$. Mature multinucleated osteoclasts are short lived, with an active life span of approximately 3 days in mice (24), indicating that overt osteolytic activity is strictly restricted in vivo. Because the ER stress sensors are one of the key molecules in deciding cell survival and apoptosis, we asked whether the UPR, specifically the IRE1 $\alpha$ /XBP1 pathway, is involved in osteoclast differentiation or in the regulation of apoptosis.

In the present study, we unexpectedly found that the IRE1 $\alpha$ / $\mathrm{XBP1}$ pathway is transiently activated during osteoclastogen- 
A

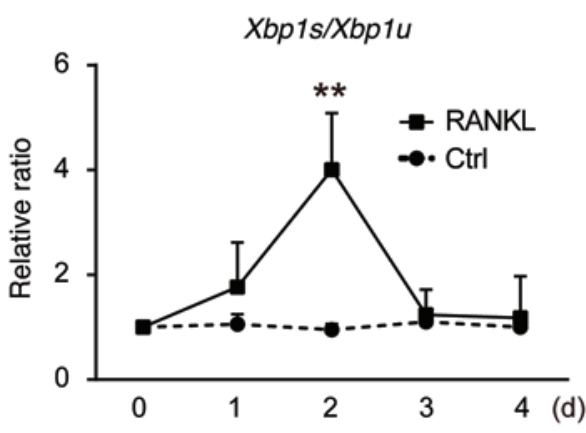

B

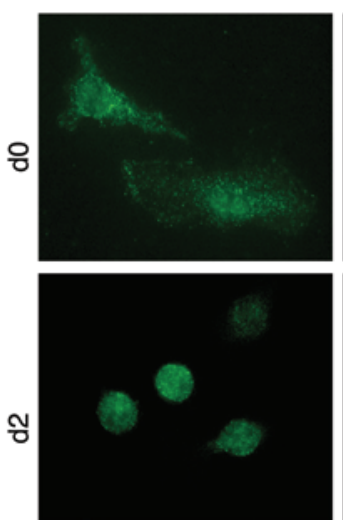

DAPI
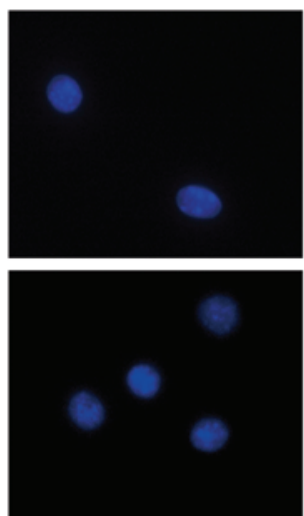

Merged

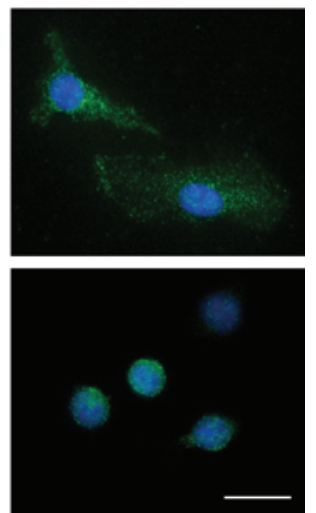

Figure 1. The IRE1 $\alpha / X B P 1$ pathway is activated in BMMs after RANKL stimulation. (A) Time-course analysis of the $X b p 1 s / X b p 1 u$ transcript ratio in BMMs treated with CSF1 alone (Ctrl) or with CSF1 and SRANKL (50 ng/ml) (RANKL). $n=4$ replicates. Values represent mean \pm SD. ${ }^{*} P<0.005$. The $X b p 1 s / X b p 1 u$ ratio on day 1 is set to 1 . Statistical analysis was performed using Student's $t$ test. (B) BMMs treated with sRANKL for 0 or 2 days were immunostained for XBP1 and counterstained with DAPI. Scale bar: $20 \mu \mathrm{m}$.

esis and that the abrogation of this pathway markedly suppresses osteoclast formation in vitro. In accordance, the conditional inactivation of IRE1 $\alpha$ in BM cells resulted in reduced osteoclast numbers and, consequently, higher bone volume in vivo. Notably, we found that XBP1 functions as a transcription factor for the $N$ fatc1 gene, the master regulator of osteoclastogenesis. In addition, we show that the activation of IRE1 $\alpha$ is triggered indirectly by $\mathrm{Ca}^{2+}$ oscillation mediated by inositol 1,4,5-trisphosphate receptors 2 and 3 (ITPR2 and ITPR3), the $\mathrm{Ca}^{2+}$ channel proteins located in the ER (25), during osteoclastogenesis. These results reveal that the UPR-mediated by the IRE1 $\alpha / \mathrm{XBP} 1$ pathway serves as a positive regulator of osteoclast differentiation by promoting $\mathrm{Nfatc1}$ transcription and suggest that the IRE1 $\alpha / \mathrm{XBP} 1$ pathway is a potential target for treating pathological bone resorption.

\section{Results}

The UPR is induced during osteoclastogenesis. To assess the potential involvement of the IRE1 $\alpha / \mathrm{XBP} 1$ pathway in osteoclastogenesis or in the maintenance of mature osteoclasts, we first asked whether the UPR is induced during osteoclast differentiation. We performed an in vitro osteoclast formation assay using WT BM macrophages (BMMs), recombinant soluble RANKL (sRANKL), and murine CSF1. Interestingly, we found that the expression level of the Xbp1s transcript, which reflects the activity of IRE1 $\alpha$, transiently increases in BMMs undergoing differentiation (Figure 1A). The ratio of $X b p 1 s / X b p 1 u$ (unspliced $X b p 1$ transcript) peaked 2 days after sRANKL treatment and subsequently decreased to almost basal levels. No changes in the Xbp1s/Xbp1u transcript ratio were observed in BMMs treated with CSF1 alone. UPR induction in the sRANKL-treated BMMs was also confirmed by the nuclear accumulation of the transcription factor XBP1s (Figure 1B) and by the increased expression of Atf4, Atf6, Hspa5 (encodes heat shock $70 \mathrm{kDa}$ protein 5 or BiP), and Eif2ak3 (encodes PERK) transcripts (Supplemental Figure 1; supplemental material available online with this article; doi:10.1172/JCI76765DS1). These observations show that ER stress is transiently induced during osteoclast differentiation under physiological conditions.
The IRE1 /XBP1 pathway promotes osteoclast differentiation. The transient increase in the expression of $X b p 1 s$ suggests that the IRE1 $\alpha / \mathrm{XBP} 1$ pathway potentially participates in the regulation of osteoclast differentiation. To test this hypothesis, we used siRNA-mediated gene silencing to suppress the IRE1 $\alpha / \mathrm{XBP} 1$ pathway in WT BMMs and subjected these cells to an in vitro osteoclast formation assay. The depletion of Ern1 (encodes IRE1 $\alpha$ ) by siRNA in the WT BMMs significantly suppressed the formation of tartrate-resistant acid phosphatase-positive (TRAP-positive) osteoclasts compared with control siRNA-treated BMMs (Figure 2A). Furthermore, $X b p 1$ gene silencing showed results nearly identical to those of Ern1, indicating that suppression of osteoclast formation by siRNA against Ern1 is XBP1 dependent. Suppression of the transcripts for Ern1 and Xbp1 in siRNA-transfected BMMs was confirmed by quantitative PCR (Supplemental Figure 2). This observation was also reproduced using a different set of siRNAs against Ern1 and Xbp1 (data not shown), and using the osteoclast progenitor-like cell line RAW264.7 (Supplemental Figure 3). Reduced bone resorption activity in Xbp1 siRNA-treated BMMs was confirmed by pit assay (Figure $2 \mathrm{~B}$ ). In addition, we found that the pharmacological inhibition of the IRE1 $\alpha$ endonuclease using STF-083010 (26) significantly suppressed osteoclast formation (Figure 2C). To evaluate the participation of the IRE1 $\alpha /$ XBP1 pathway in a more relevant setting, we next generated Ern1 $1^{f l o x} / f l o x / M x 1-$ Cre (henceforth referred to as ErnI ${ }^{M x 1}$ ) mice in which Cre recombinase was expressed under the control of the IFN-inducible $M x 1$ promoter $(27,28)$ to deplete IRE1 $\alpha$ from BM cells. Ten-day-old control and $E r n 1^{M x 1}$ mice were injected i.p. with polyinosinic-polycytidylic acid (pIpC) 3 times at 2-day intervals and were analyzed when they reached 9 weeks of age. The BMMs collected from the $E r n 1^{M x 1}$ mice were incapable of processing Xbp1 transcripts, even in the presence of thapsigargin, a potent inducer of ER stress (Figure 2D), indicating that IRE1 $\alpha$ activity was completely lost in these cells, whereas the processing of $X b p 1$ transcripts remained intact in BMMs collected from Ern1 ${ }^{M x 1}$ mice not treated with pIpC. In support of the results of the gene-silencing experiments, BMMs lacking IRE1 $\alpha$ formed significantly fewer TRAP-positive osteo- 
A

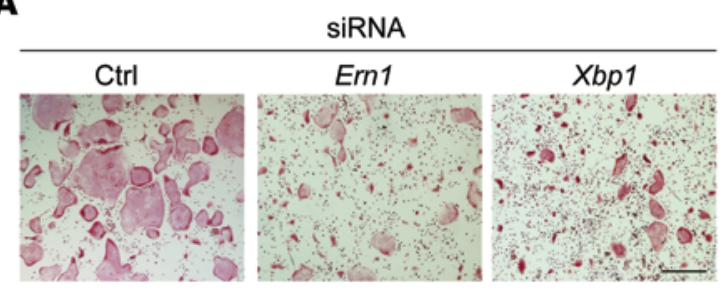

C

$(-)$

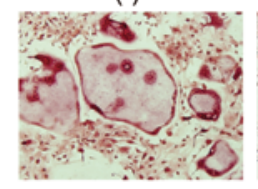

E

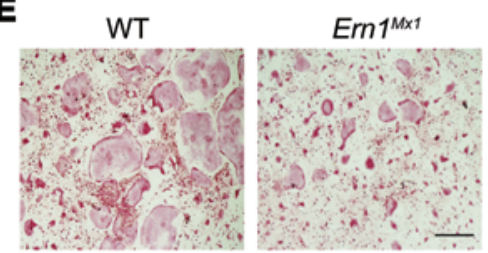

STF-083010

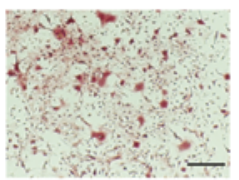

D

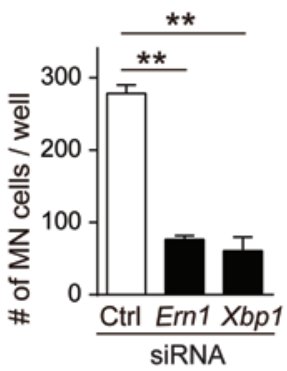

B
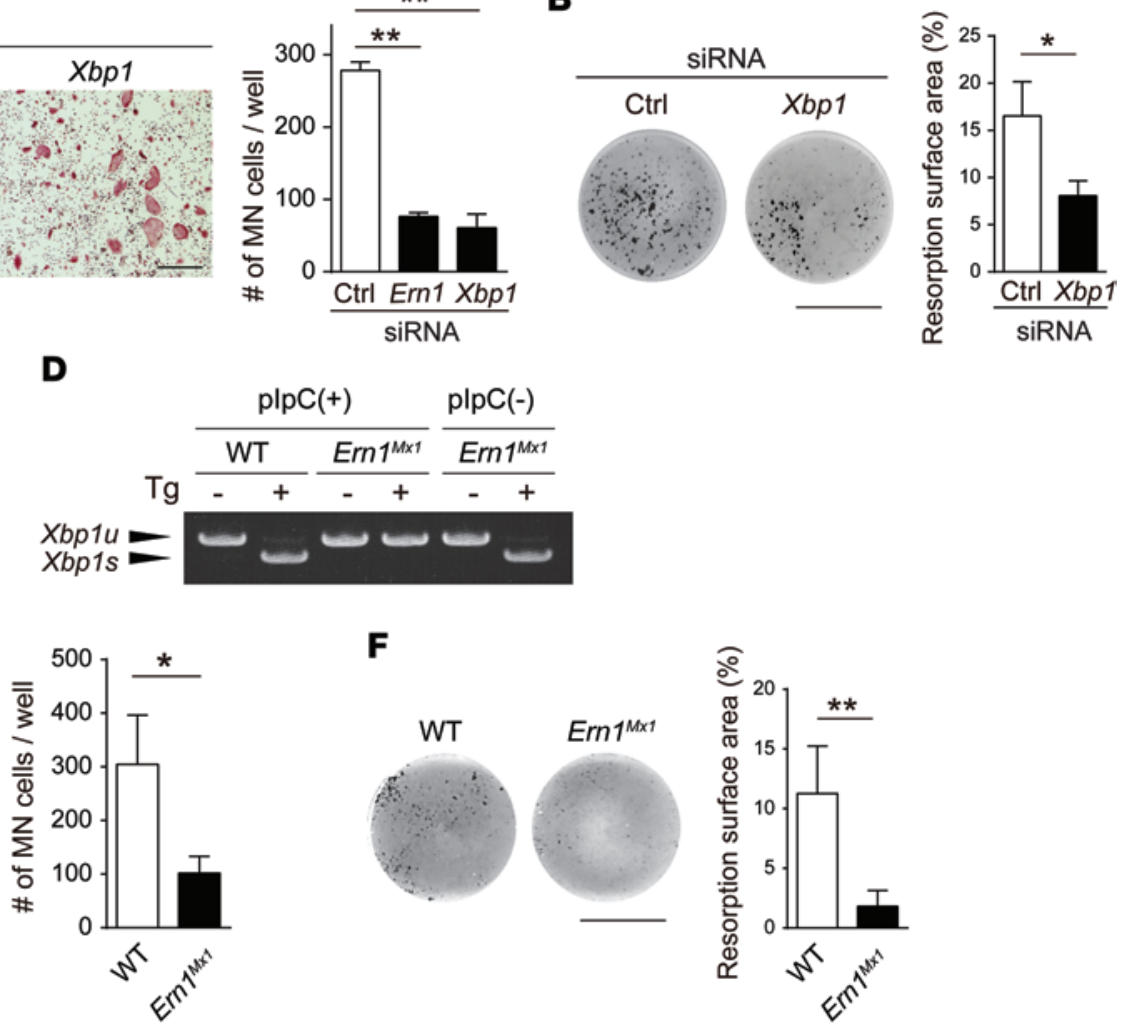

$\mathbf{F}$

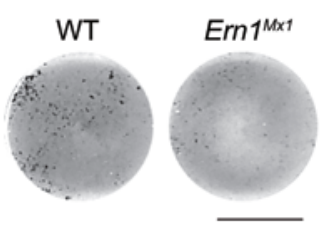

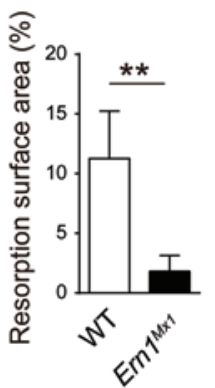

Figure 2. Abrogation of the IRE1 / /XBP1 pathway suppresses osteoclast formation. (A) WT BMMs were transfected with control, Ern1, or Xbp1 siRNA and incubated with recombinant sRANKL and CSF1 for 4 days. Cells were stained with TRAP, and the number of TRAP-positive multi-nucleated (MN) cells was recorded. Representative photomicrographs of TRAP-stained cells in each well are presented. Scale bar: $200 \mu \mathrm{m}$. $n=3$ replicates. Values represent mean \pm SD. ${ }^{* *} P<0.005$. (B) Pit formation assay using WT BMMs transfected with control or Xbp1 siRNA. Representative images of osteoclastformed pits (left panel) and the surface ratio of the pits (right panel) are shown. $n=3$ replicates. Values represent mean \pm SD. ${ }^{*} P<0.05$. Scale bar: 5 mm. (C) Representative photomicrographs of TRAP-stained BMMs treated with vehicle (-) or STF-083010 (30 $\mu$ M). Scale bar: $200 \mu \mathrm{m}$. (D) Reverse-transcription-PCR analysis of Xbp1s and Xbp1u transcripts. BMMs were collected from plpC-treated WT and Ern ${ }^{M \times 1}$ mice and nontreated Ern ${ }^{M \times 1}$ mice, and were incubated with thapsigargin (Tg) $(1 \mu \mathrm{M})$. (E) BMMs collected from WT and Ern7 ${ }^{\mathrm{M} \times 1}$ mice were incubated with sRANKL and CSF1 for 4 days. Cells were stained for TRAP, and the number of TRAP-positive multinucleated cells was recorded (right panel). Representative photomicrographs of TRAP-stained cells in each well are presented. Scale bar: $200 \mu \mathrm{m} . n=3$ replicates. Values represent mean \pm SD. ${ }^{*} P<0.05$. (F) Pit formation assay using BMMs collected from WT and Ern $7^{M \times 1}$ mice. Representative images of osteoclast-formed pits (left panel) and the surface ratio of the pits (right panel) are shown. $n=3$ replicates. Values represent mean $\pm \mathrm{SD}$. ${ }^{* *} P<0.005$. Scale bar: $5 \mathrm{~mm}$. Statistical analysis was performed using Student's $t$ test.

clasts and showed significantly lower bone resorption activity compared with the WT BMMs (Figure 2, E and F). These observations show that the IRE1 $\alpha / \mathrm{XBP} 1$ pathway is critically involved in the regulation of osteoclast differentiation and that the abrogation of this pathway markedly suppresses osteoclast formation in vitro.

Conditional inactivation of IRE1 $\alpha$ results in increased bone mass. The Ern $1^{M x l}$ mice treated with pIpC showed no overt anomalies; however, $\mu \mathrm{CT}$ analysis of the femurs revealed that the Ern1 ${ }^{M x 1}$ mice exhibited significantly higher bone mass compared with their WT littermates, as highlighted by the increase in the bone volume/total volume and the trabecular number (Figure 3, A and B). Histomorphological analysis showed an increase in bone volume and a decrease in the number of osteoclasts as well as markers of bone resorption in the $E r n 1^{M x l}$ mice compared with the WT animals (Figure 3, C and D). Although the expression levels of the $E r n 1$ transcript in osteoblasts were reduced in $E r n 1^{M x 1}$ mice compared with the WT animals, the reduction was moderate $(\sim 27 \%)$ and there were no significant changes in the expression levels of osteoblastic markers in Ern1 ${ }^{M x I}$ mice compared with the WT con- trols (Supplemental Figure 2A). In accordance, no significant differences were found in bone formation indices in the $E r n 1^{M x l}$ mice compared with the WT mice (Supplemental Figure 2, B and C). These observations indicate that the increase in bone volume in $E r n 1^{M x l}$ mice was primarily derived from defective osteoclastogenesis, but not from increased bone formation.

The decreased osteoclast number in the Ern ${ }^{M x l}$ mice may also be derived from the defects in the development of osteoclast precursors in the absence of IRE1 $\alpha$ in BM cells; therefore, we also evaluated the population of monocytes/macrophages $\left(\mathrm{Gr}-\mathrm{I}^{+} \mathrm{CD} 11 \mathrm{~b}^{+}\right)$and osteoclast precursors $\left(\mathrm{CD} 117^{+} \mathrm{CD} 11 \mathrm{~b}^{\text {dull }} \mathrm{CD} 115^{+}\right)(29)$ in the BM. Although, there was an increase in the absolute number of osteoclast precursors in $E r n 1^{M x l}$ mice ( $49 \%$ ), no apparent difference was observed in the overall population ratio of these cells between the Ern1 ${ }^{M x 1}$ and WT mice (Figure 4, A and B). Furthermore, the lack of IRE1 $\alpha$ did not affect cell survival or growth, as assessed by evaluations of the MTT-based assay and caspase- $3 / 7$ activities (which reflect the amount of cells undergoing apoptosis) (Figure 4, C and D). Therefore, we concluded that the decreased osteoclast number and increased bone mass in 
A

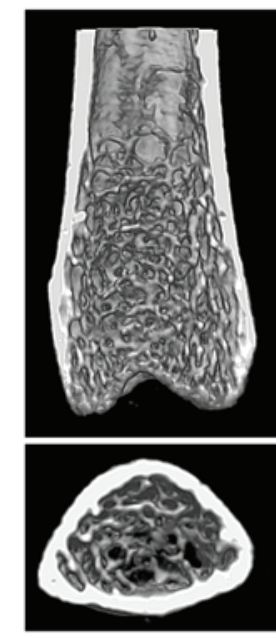

D

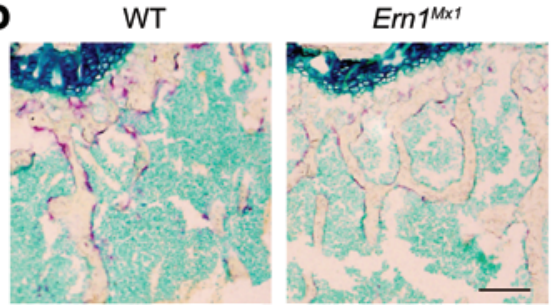

B

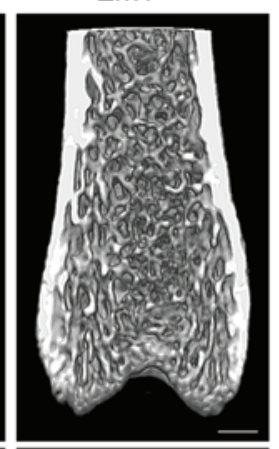

c
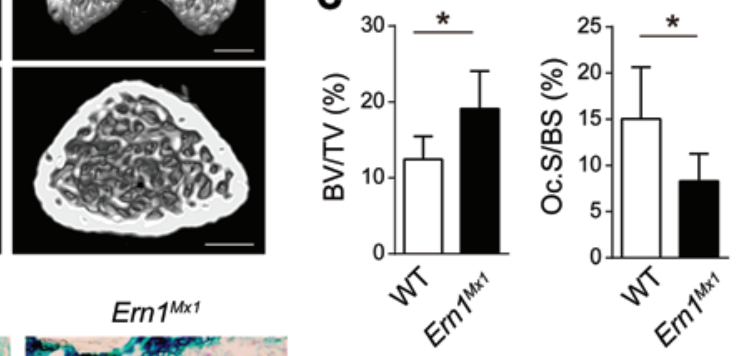
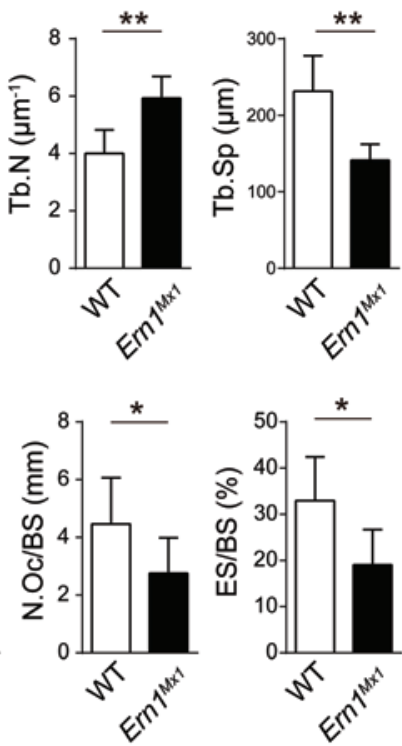

Figure 3. Conditional inactivation of IRE1 $\alpha$ results in increased bone volume and decreased osteoclast number in vivo. (A) Reconstituted 3D images of the distal femur. Scale bars: 0.5 mm. (B) $\mu$ CT-based structural analysis of the femur ( $n=6$ mice/group). BV/TV, bone volume/tissue volume; Tb.Th, trabecular thickness; Tb.N, trabecular number; Tb.Sp, trabecular space. Values represent mean \pm SD. ${ }^{* *} P<0.005$. (C) Histomorphometric analysis of the tibial metaphysis ( $n=7$ mice/ group). ES/BS, erosion surface/bone surface; Oc.S/BS, osteoclast surface/bone surface; N.Oc/BS, osteoclast number/bone surface. Values represent mean \pm SD. ${ }^{*} P<0.05$. (D) Sections of the tibial metaphysis stained for TRAP. Scale bar: $200 \mu \mathrm{m}$. Statistical analysis was performed using Student's $t$ test.

the $E r n 1^{M x 1}$ mice primarily resulted from defective osteoclastogenesis and bone resorption and that the absence of IRE1 $\alpha$ does not have a significant impact on the growth or survival of osteoclast precursors.

Chimeric mice lacking IRE1 $\alpha$ in BM cells show higher bone mass and decreased osteoclastic activity. Since $M x 1$ promoter activity is not restricted to $\mathrm{BM}$ cells but is also observed in a wide range of tissues (28), the increased bone volume in $E r n 1^{M x 1}$ mice could have derived from other factors that are not related to defective osteoclastogenesis. To address this issue, we performed BM transplantation experiments. BM cells were collected from 8- to 10-weekold Ern $1^{M x 1}$ or WT mice treated with pIpC. The cells were injected into lethally irradiated 8-week-old WT mice. The mice were maintained for 12 weeks before analysis (the protocol is outlined in Figure 5A). Although there was a decrease in absolute bone volume due to irradiation (30), $\mu \mathrm{CT}$ analysis showed that the mice reconstituted with $\mathrm{BM}$ cells isolated from $E r n 1^{M x 1}$ mice had higher bone mass compared with those reconstituted with WT BM cells, as illustrated by the increase in the bone volume/total volume and trabecular number (Figure 5B). Most importantly, bone morphometric analysis revealed a significant decrease in the osteoclast number and bone resorption activity in the mice reconstituted with BMMs lacking IRE1 $\alpha$ compared with those reconstituted with WT cells (Figure 5C). These observations further strengthen the idea that higher bone mass in the $E r n 1^{M x 1}$ mice was due to defective osteoclastogenesis in BM cells lacking IRE1 $\alpha$.
BMMs lacking IRE1 1 are defective in inducing Nfatc1 after $R A N K L$ stimulation. Given that IRE1 $\alpha$-deficient BMMs are defective in forming osteoclasts, we next determined whether the absence of IRE1 $\alpha$ affects the expression of osteoclastogenesisrelated transcription factors, including $N f a t c 1$, Fos (encodes FBJ murine osteosarcoma viral oncogene homolog or c-Fos), Jund (encodes Jun D protooncogene), Rela (encodes V-Rel reticuloendotheliosis viral oncogene homolog or nuclear factor $\mathrm{NF}-\mathrm{\kappa B}$ P65 subunit), and Spi1 (encodes PU.1). Notably, gene-expression analysis revealed that the BMMs lacking IRE1 $\alpha$ expressed significantly less $N f a t c 1$ after RANKL stimulation compared with the control cells; however, no significant differences were observed for the other transcription factors (Figure 6A). There were no differences in the expression levels in any of these genes before RANKL stimulation. The decrease in Nfatc1 transcript but not Fos was also observed following the inhibition of IRE1 $\alpha$ endonuclease activity using STF-083010 (Figure 6B) and after the depletion of $X b p 1$ in WT BMMs using siRNA (Figure 6C). The decrease in the transcript levels of NFATc1 target genes Ctsk (encodes Cathepsin K) and Acp5 (encodes TRAP) in IRE1 $\alpha$-deficient BMMs was also confirmed (Figure 6D). These observations suggest that the abrogation of the IRE1 $\alpha / \mathrm{XBP} 1$ pathway results in the suppression of $N f a t c 1$ transcription independently of other osteoclastogenesis-related transcription factors and thereby inhibits osteoclast differentiation. 
A

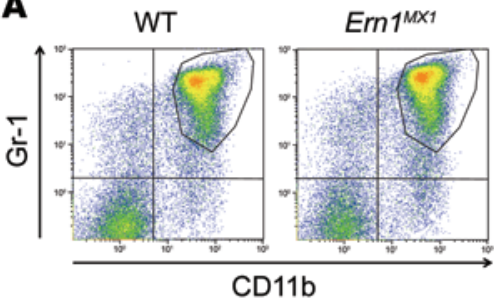

B

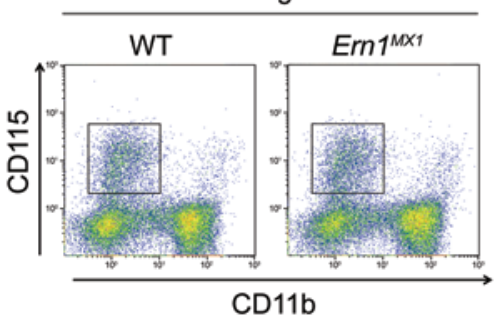

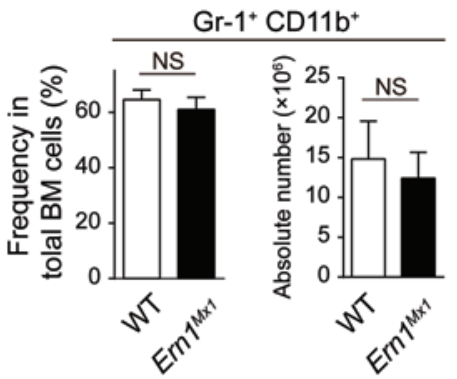

C

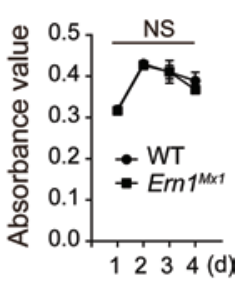

D

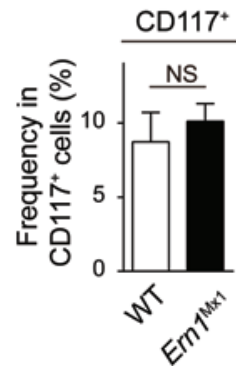

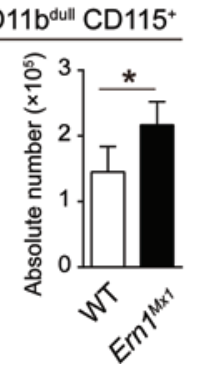

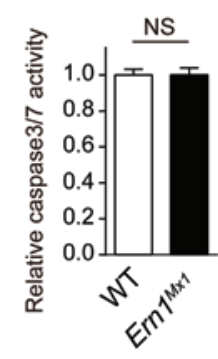

Figure 4. Lack of IRE1 $\alpha$ activity does not affect cell survival of BMMs or population of osteoclast precursors. (A and B) Flow cytometric analysis of the monocyte/macrophage (A) and osteoclast precursor (B) populations. Representative flow cytometry plots for each experiment are shown (left panels). Frequency in total BM cells (A) or in CD117+-gated BM cells (B) and absolute number of these cells (in bilateral femurs and tibiae) are presented (right panels). $n=4$ for WT and $n=8$ for $E r n 7^{M \times 1}$ mice. Values represent mean $\pm S D .{ }^{*} P<0.05$. (C) Cell survival rate of WT and $E r n 7^{M \times 7}$ BMMs incubated with sRANKL for 1 to 4 days was evaluated using an MTT-based assay. $n=3$ replicates. (D) Caspase-3/7 activity in the WT and $E r n 7^{M \times 1}$ BMMs treated with SRANKL was evaluated. $n=3$ replicates. Values represent mean \pm SD. Statistical analysis was performed using Student's $t$ test.
$X B P 1 s$ binds to the promoter of the Nfatc1 gene and promotes $N$ fatc1 transcription. Based on a potential link between the IRE1 $\alpha$ / XBP1 pathway and $N f a t c 1$, we next sought to investigate how XBP1s affected the regulation of $N f a t c 1$. The promoter region of the Nfatc1 gene bears 2 binding sites for NFATc1 (5'-TTTCC-3') through which the transcription of $N f a t c 1$ is amplified in a positive-feedback manner. Interestingly, nucleotide sequence analysis revealed 2 UPR element-like sequences (5'-GGAAG-3') (8) located adjacent to (less than $10 \mathrm{bp}$ apart) and flanking the tandem NFATc1-binding motifs (Figure 7A). This finding prompted us to investigate whether XBP1s acts as a transcription factor for Nfatc1. To test this possibility, we performed ChIP assays using nuclear extracts from sRANKL-treated and nontreated WT BMMs and a set of primers designed to amplify the promoter region of Nfatc1(-764 to $-551 \mathrm{bp}$ ) (Figure 7A). The PCR amplification of the ChIP revealed that both XBP1 and NFATc1, the latter of which was used as a positive control for the assay, were capable of binding to the $N$ fatcl promoter (Figure 7B). As expected, positive amplification was obtained only in the sRANKL-treated but not in nontreated cells. A set of primers outside of the NFATc1-binding sites ( -942 to -811 bp) obtained no positive signal. Identical results were observed in RAW264.7 cells (data not shown).

Based on these observations, we generated a luciferase reporter vector ( $\mathrm{ffatc1-800)}$ harboring the promoter region of the $N f a t c 1$ gene ( -800 to $+1 \mathrm{bp}$ ) and performed luciferase reporter assays using the XPB1s and NFATc1 expression vectors. A significant increase in luciferase activity was observed when either the XPB1s or the NFATc1 expression vector was cotransfected with the reporter plasmid, and the concomitant expression of both XPB1s and NFATc1 showed an additive effect on luciferase activity (Figure 7C). The removal of either of the putative XBP1sbinding sites (Nfatc1-800 $\Delta-697-693$ and $N f a t c 1-800 \Delta-667-663$ ) rendered the reporter construct unresponsive to XBP1s; however, the transcriptional activity induced by NFATc1 remained intact (Figure 7C). These observations suggest that defective osteoclastogenesis in BMMs lacking IRE1 $\alpha$ is derived from insufficient expression of NFATc1 after RANKL stimulation. To confirm this hypothesis, we next asked whether forced expression of NFATc1 in IRE1 $\alpha$-deficient BMMs can rescue the expression of $\mathrm{Nfatc1-}$ dependent genes. WT and IRE1 $\alpha$-deficient BMMs were transfected with NFATc1 expression vector or GFP expression vector and incubated in the presence of SRANKL and CSF1 for 3 to 4 days. As shown in Figure 7D, we found a significant increase in the levels of Ctsk and Acp 5 transcripts in IRE1 $\alpha$-deficient BMMs transfected with NFATc1 expression vector compared with those transfected with GFP. These data indicate that defective osteoclastogenesis in IRE1 $\alpha$-deficient BMMs is primarily derived from insufficient induction of $N$ fatcl after RANKL stimulation.

The activation of IRE1 $\alpha$ during osteoclastogenesis is dependent on $\mathrm{Ca}^{2+}$ influx mediated by ITPR2 and ITPR3. The transient activation of the IRE1 $\alpha / \mathrm{XBP} 1$ pathway during osteoclastogenesis (Figure 1) suggests that ER stress is induced after RANKL stimulation in BMMs. Because the RANKL-induced ER stress in BMMs appeared unlikely to be derived from protein synthesis overload, we hypothesized that other mechanisms were involved in eliciting the UPR. RANKL-RANK signaling triggers $\mathrm{Ca}^{2+}$ influx from the intracellular storage sites in the ER primarily through ITPR2 and ITPR 3 and activates the $\mathrm{Ca}^{2+}$-dependent phosphatase calcineurin $(23,25,31)$, which is essential for the activation of the transcription factor NFATc1. The fluctuation of $\mathrm{Ca}^{2+}$ levels in the ER lumen results in ER stress $(32,33)$, which raises the possibility that $\mathrm{Ca}^{2+}$ outflow from the ER lumen triggered by RANKL-RANK signaling indirectly activates IRE1 $\alpha$. To test this idea, we asked whether the stimulation of ITPRs using ATP, an agonist for phospholipase C-coupled P2Y receptor $(34,35)$, induces ER stress in BMMs and activates IRE1 $\alpha$. As anticipated, ATP treatment evoked $\mathrm{Ca}^{2+}$ outflow from the ER and concomitantly induced the processing of Xbp1 transcripts (Figure 8, A and B). Furthermore, we found that the sRANKL-induced processing of the $X b p 1$ transcripts was significantly reduced in Itpr $2^{-/-}$Itpr $3^{-/-}$BMMs compared with the WT BMMs (Figure 8C). The pharmacological inhibition of ITPRs by 2 -aminoethoxydiphenyl borinate, a potent inhibitor of ITPRs, 
A

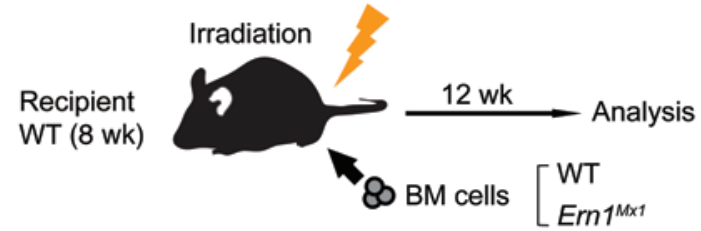

B
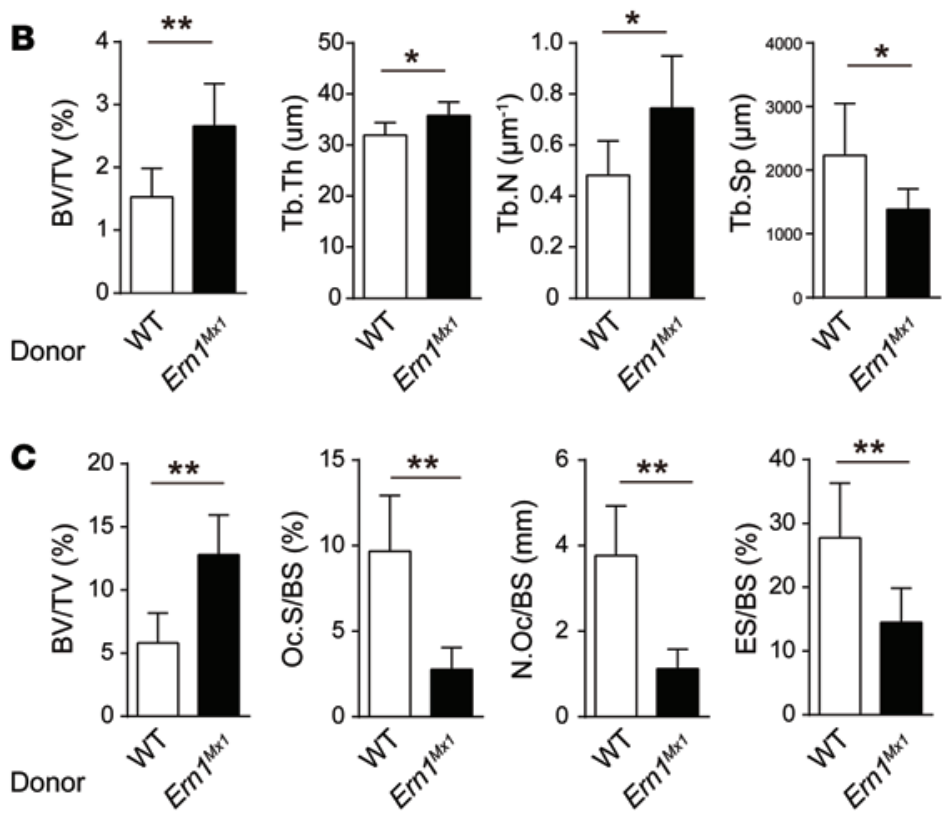

showed similar results (Figure 8D). Taken together, these results show that the activation of the IRE1 $\alpha /$ XBP1 pathway during osteoclastogenesis is, at least in part, indirectly induced by $\mathrm{Ca}^{2+}$ outflow from the ER lumen through ITPR2 and ITPR3.

\section{Discussion}

Our data show that ER stress is induced during osteoclast differentiation and that the abrogation of the UPR mediated by the IRE1 $\alpha$ / XBP1 pathway markedly suppresses the formation of multinucleated osteoclasts. In accordance, we found that the conditional inactivation of IRE1 $\alpha$ in vivo results in a substantial increase in bone volume due to defective osteoclastic bone resorption. Most importantly, we found that the promoter region of the $\mathrm{Nfatc1}$ gene bears potential binding sites for XBP1s and that XBP1s induces transcription of $\mathrm{Nfatc1}$ by directly binding to these sites. The present study reveals what we believe to be a novel intracellular pathway that links UPR and Nfatcl transcription and an unconventional role for the IRE1 $\alpha / \mathrm{XBP} 1$ pathway as a crucial regulator of osteoclast differentiation (Figure 9).

One of the important findings of the present study may be the potential involvement of $\mathrm{Ca}^{2+}$ influx mediated by ITPRs in inducing the UPR during osteoclastogenesis. Although we cannot fully exclude other factors such as protein synthesis overload and the generation of reactive oxygen species as a cause for ER stress, our observations indicate that the activation of the IRE1 $1 /$ XBP1 pathway during osteoclastogenesis is, at least in part, dependent on $\mathrm{Ca}^{2+}$ oscillation mediated by ITPR2 and ITPR3. In support of this finding, BMMs lacking ITPR2 and ITPR3 exhibited diminished $\mathrm{Ca}^{2+}$ oscillation during osteoclastogenesis and were defective in form-
Figure 5. Chimeric mice lacking IRE1 $\alpha$ in BM cells exhibit higher bone volume and decreased osteoclast number. (A) Schema showing the protocol for BM transplantation experiment. (B) $\mu \mathrm{CT}$ based structural analysis of the femur ( $n=7$ mice/group). Values represent mean $\pm \mathrm{SD}$. ${ }^{*} P<0.05$; ${ }^{* *} P<0.005$. (C) Histomorphometric analysis of the tibial metaphysis ( $n=7$ mice/group). Values represent mean $\pm \mathrm{SD} .{ }^{* *} P<0.005$. Statistical analysis was performed using Student's $t$ test.

ing osteoclasts in vitro (31). Our data suggest that $\mathrm{Ca}^{2+}$ oscillation elicited by RANKL-RANK signaling indirectly induces ER stress and activates the IRE1 $\alpha / \mathrm{XBP1}$ pathway, which in turn promotes $\mathrm{Nfatc1}$ transcription. The present study proposes a model that $\mathrm{Ca}^{2+}$ oscillation mediates the activation of 2 different (IRE1 $\alpha$ - and calcineurin-dependent) pathways in inducing $\mathrm{Nfatc1}$ transcription during the early stage of osteoclast differentiation. In contrast, ITPR2 and ITPR3 are dispensable for osteoclast formation in vivo (31) and a lower level of Xbp1 transcripts is still processed in the absence of ITPR2 and ITPR3 (Figure 8C); therefore, it is likely that other $\mathrm{Ca}^{2+}$ channels or different mechanisms that are not related to $\mathrm{Ca}^{2+}$ influx are also involved in the induction of ER stress after RANKL stimulation. In addition, the observation that ER stress is only transiently induced after RANKL stimulation (Figure 1A) indicates that the induction of XBP1s is required temporarily to trigger the autoamplification of NFATc1 during osteoclastogenesis and that the UPR has a limited contribution in maintaining the functions of mature osteoclasts.

The involvement of the UPR in osteoblasts and chondrocytes has been widely explored (11, 15, 36-38). Because both osteoblasts and chondrocytes produce a large amount of extracellular matrix proteins and have a highly developed ER, it is conceivable that these cells are constitutively under ER stress and that the UPR has indispensable roles in maintaining their functions. Intriguingly, past studies revealed that the UPR in osteoblasts not only functions to alleviate ER stress, but also positively regulates osteoblast differentiation through enhancing osteoblast-related genes $(11,36,37)$. We previously showed that the UPR is elicited in BMP2-induced osteoblast differentiation, presumably due to protein synthesis overload, and that XBP1s promotes $S p 7$ transcription (encodes the transcription factor Osterix), one of the master regulators of osteoblast differentiation (11). The reason for the lack of any significant changes in osteoblastic indices in the $E r n 1^{M x l}$ mice is not clear; however, it is most likely due to an insufficient abrogation of IRE1 $\alpha$ in osteoblasts (Supplemental Figure 4). These observations, in addition to the results of BM transplantation experiments (Figure 5), are consistent with the notion that the increased bone volume in the $E r n 1^{M x l}$ mice was derived from decreased osteoclastic bone resorption, but not from increased bone formation.

There are limitations in the present study. First, bone morphometric and $\mu \mathrm{CT}$ analyses were performed exclusively using male mice, and we cannot fully exclude the possibility that lack of IRE1 $\alpha$ in BM cells may affect female mice differently than male mice. Second, all animal experiments were done under unchallenged conditions, and therefore, further studies are mandatory to clarify whether the IRE1 $\alpha / \mathrm{XBP} 1$ pathway is involved in the development of disorders with increased osteoclastic bone resorption. Finally, 

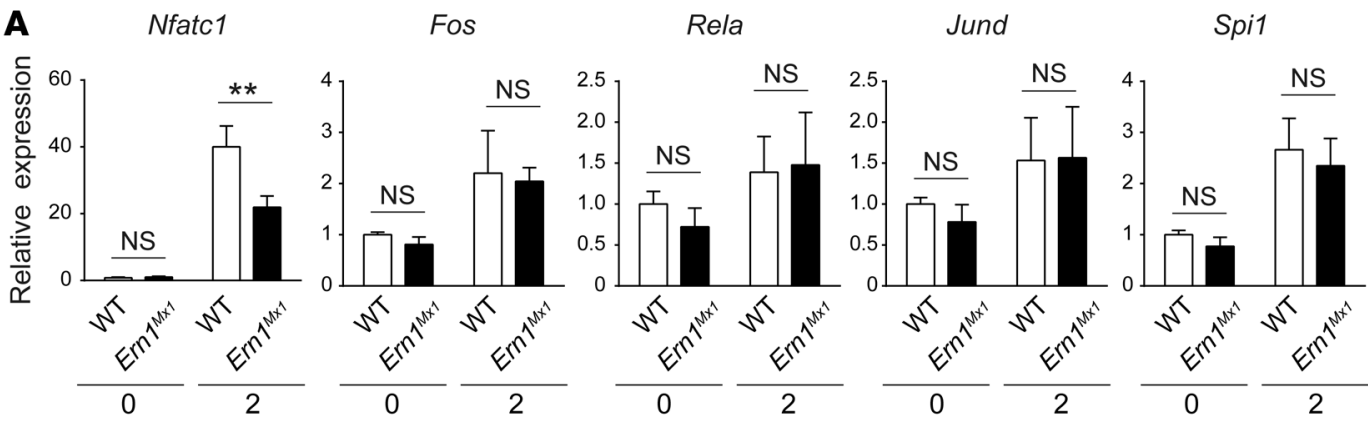

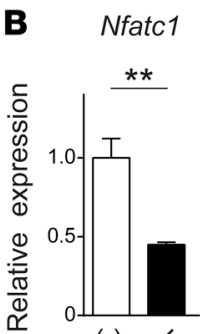

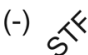
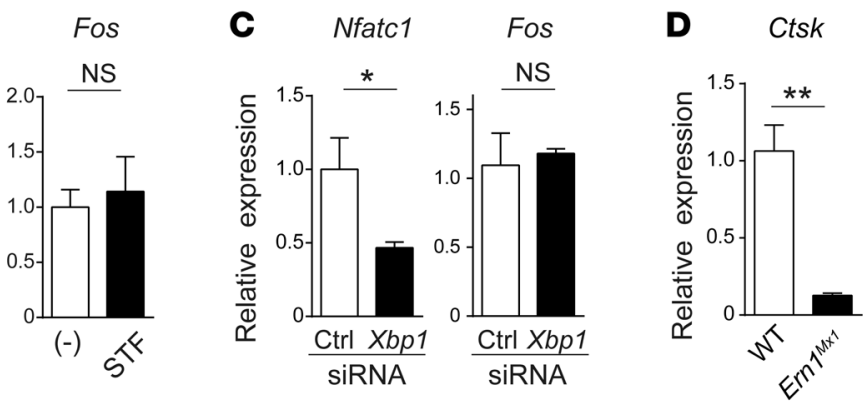

Acp5

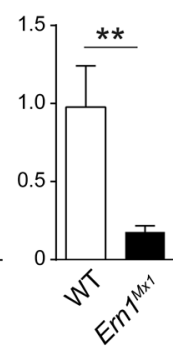

Figure 6. Disruption of the IRE1a/XBP1 pathway suppresses Nfatc1 expression. (A) Relative transcript expression levels of $\mathrm{Nfatc1,} \mathrm{Fos,} \mathrm{Rela,} \mathrm{Jund,} \mathrm{and}$ Spi1 in WT and Ern ${ }^{M \times 1}$ BMMs treated with sRANKL for 0 or 2 days. $n=3$ replicates. Values represent mean \pm SD. ${ }^{* *} P<0.005$. (B and C) WT BMMs were treated the vehicle (-) or STF-083010 (STF) (B) or with control or Xbp1 siRNA (C) and incubated in the presence of recombinant sRANKL for 2 days. The expression levels of $N f a t c 1$ and Fos transcripts were quantitatively analyzed. $n=3$ replicates. Values represent mean \pm SD. ${ }^{*} P<0.05 ;{ }^{* *} P<0.005$. (D) Relative transcript expression levels of NFATc1 target genes Ctsk and Acp5 in WT and Ern ${ }^{1 \times 1}$ BMMs treated with sRANKL for 3 days. $n=3$ replicates. Values represent mean \pm SD. ${ }^{* *} P<0.005$. Statistical analysis was performed using Student's $t$ test.

the reason for the increase in the absolute number of osteoclast precursors in $E r n 1^{M x 1}$ mice remains elusive (Figure $4 \mathrm{~B}$ ). Since the frequency of osteoclast precursors in $\mathrm{CD}_{117^{+}} \mathrm{BM}$ cells was comparable between WT and Ern ${ }^{M x 1}$ mice, this result indicates that there was an increase in the absolute number of $\mathrm{CD} 117^{+} \mathrm{BM}$ cells in $E r n 1^{M x 1}$ mice. This may have been derived from a homeostatic mechanism to offset the effects of defective differentiation of osteoclasts or other types of cells in $E r n 1^{M x 1}$ mice. Nevertheless, it is also clear from this observation that the decreased osteoclast number in $E r n 1^{M x 1}$ mice was not due to a decrease in the number of osteoclast precursors in BM cells lacking IRE1 $\alpha$.

In conclusion, the present study revealed an unexpected role of the IRE1 $\alpha / \mathrm{XBP} 1$ pathway induced by physiological ER stress as a critical regulator to promote osteoclast differentiation by enhancing Nfatc1 transcription. The IRE1 $\alpha / \mathrm{XBP} 1$ pathway is often activated in cancers and has recently emerged as a potential molecular target for anticancer therapy $(1,5,39-43)$, especially for multiple myeloma, which is characterized by malignant plasma cells producing a high amount of immunoglobulin $(26,42,44,45)$. These previous studies showed that the pharmacological inhibition of IRE1 $\alpha$ endonuclease significantly suppressed myeloma tumor growth in a model of human malignant myeloma xenografts, corroborating the idea that the IRE1 $\alpha / \mathrm{XBP} 1$ pathway may serve as a promising molecular target. In addition, a recent study showed that the targeting of the IRE1 $\alpha / \mathrm{XBP} 1$ pathway is a potential treatment strategy for triple-negative breast cancer, an aggressive subtype of breast cancer that frequently metastasizes to bones $(41,46)$. Cancer dissemination and malignant myeloma often result in the development of osteolytic lesions in which osteoclasts are frequently overactivated; therefore, the inhibition of the IRE1 $\alpha$ XBP1 pathway may be beneficial in suppressing both tumor growth and pathological bone resorption in cancer patients with bone metastases.

\section{Methods}

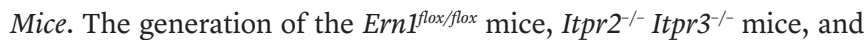
Mx1-Cre transgenic mice was previously described $(27,28,47)$. The $E r n 1^{f l o x / f l o x}$ mice exhibited no apparent pathological phenotype and were used as the WT control animals. WT and $E r n 1^{M x 1}$ mice were of mixed genetic background (129Sv, C57BL/6). For the temporal deletion of the floxed Ern1 allele in the Ern1 ${ }^{M x 1}$ mice, 10-day-old mutant and control mice were injected i.p. with $250 \mu \mathrm{g}$ of pIpC 3 times at 2-day intervals and were analyzed when they reached 9 weeks of age.

Reagents and cell lines. Anti-XBP1 (M-186) and anti-NFATc1 (7A6) antibodies were obtained from Santa Cruz Biotechnology Inc. All of the siRNAs were obtained from Sigma-Aldrich. STF083010 was purchased from Calbiochem, and sRANKL was purchased from PeproTech. The RAW264.7, 293T, and 293gp cells were obtained from the Riken Bioresource Center.

$\mu C T$ and histomorphometric analysis. All analyses were conducted using 9-week-old male mice. Femurs were excised and fixed with $75 \%$ ethanol. Two-dimensional images of the distal femurs were obtained by $\mu \mathrm{CT}$ scanning (R_mCT2, Rigaku), and 3D images were reconstructed using the 3D software TRI/3D-BON (Ratoc System Engineering). The bone mineral density of the femurs was measured by Phantom for Bone Mineral Quantity (Kyoto Kagaku). For histomorphometric analysis, the mice were injected s.c. with calcein ( $8 \mathrm{mg} / \mathrm{kg}$ body weight) 5 and 2 days before euthanasia. The tibiae were excised, fixed with $75 \%$ ethanol, embedded in glycol methacrylate resin, and sectioned into $5-\mu \mathrm{m}$ 
A

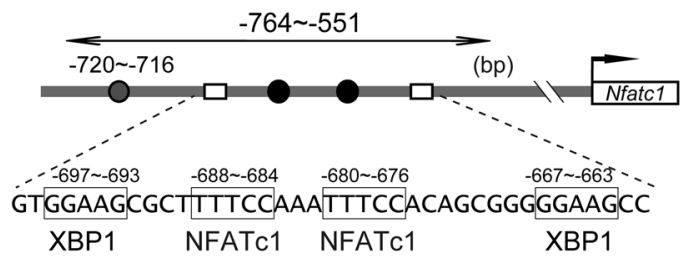

B

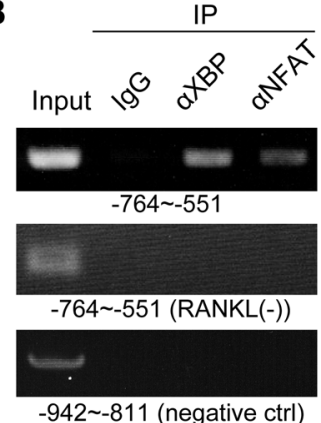

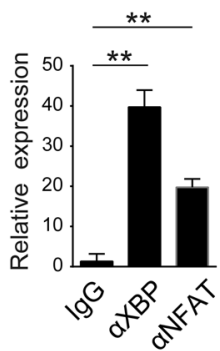

C

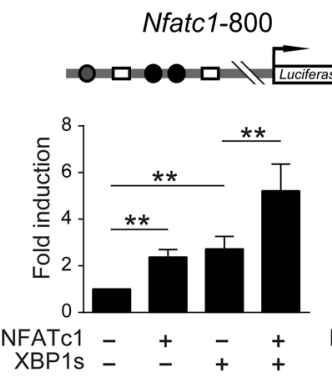

D

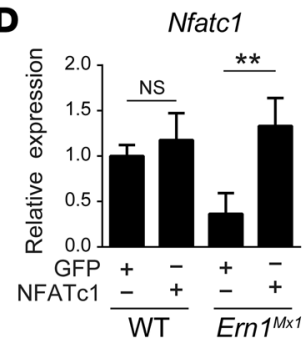

Nfatc1-800ム-697-693

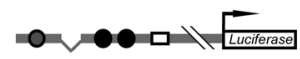

\section{Nfatc1-800 $\Delta-667-663$}
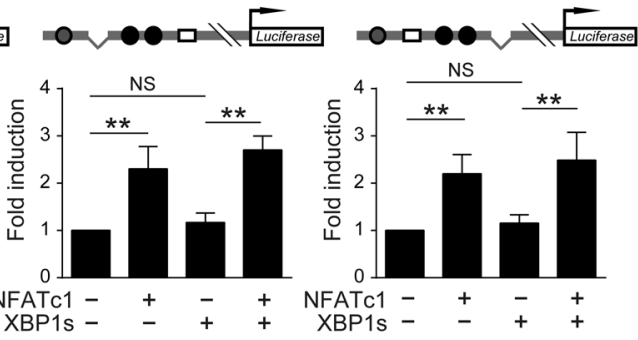

Ctsk

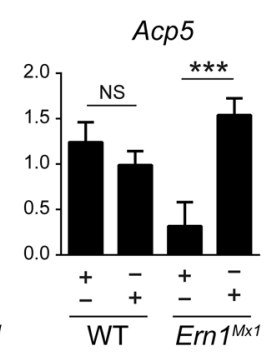

Figure 7. XBP1 binds to the promoter of the $\mathbf{N f a t c 1}$ gene and promotes its transcription. (A) A schematic of the promoter region of the Nfatc1 gene. The binding sites for NF-KB (gray circle), NFATc1 (black circles), and 2 putative XBP1s-binding sites (white rectangles) are shown. The left-right arrow indicates the expected PCR products for the ChIP assays in Figure 5B. (B) ChIP assays using control lgG, antibodies against XBP1 ( $\alpha$ XBP1), or NFATc1 ( $\alpha$ NFATc1). Cell extracts were collected from WT BMMs treated with (upper and middle panel) or without (lower panel) sRANKL. PCR of the cell extracts was performed using a set of primers either inside (-764 551) or outside (-942 811) of the promoter region containing the NFATc1-binding sites. Relative amount of the immunoprecipitated DNA fragment assessed by quantitative PCR (right panel). The amount of immunoprecipitated DNA of control lgG is set to $1 .{ }^{* *} P<0.005$. (C) Luciferase reporter assays using the WT construct (left, Nfatc1-800) or deletion mutants (middle, Nfatc1-800 $\Delta-697-693$; right, Nfatc1-800 $\Delta$-667-663) and the XPB1s and NFATc1 expression vectors. Fold induction of luciferase activity of each construct is presented. Luciferase activity of the cells transfected with reporter vector alone is set to $1 . n=3$ replicates. Values represent mean $\pm \mathrm{SD}$. ${ }^{*} P<0.005$. (D) Relative transcript expression levels of Nfatc1, Ctsk, and Acp5 in WT and Ern ${ }^{M \times 1}$ BMMs transfected with GFP or NFATc1 expression vector. BMMs transduced with the indicated vectors were incubated in the presence of CSF1 and sRANKL for 3 to 4 days to induce differentiation prior to analysis. $n=4$ replicates. Values represent mean \pm SD. ${ }^{* *} P<0.005 ;{ }^{* *} P<0.0005$. Statistical analysis was performed using Student's $t$ test.

slices. Sections were stained with toluidine blue and were subjected to histomorphometric analyses under a light microscope, using a semiautomated image analyzer (CSS-840, System Supply). The parameters for the trabecular bone were measured in an area $1.62-2.34 \mathrm{~mm}^{2}$ in size that was $1.2 \mathrm{~mm}$ above the growth plate at the proximal metaphysis. Histomorphometric analyses were performed in a blinded manner.

Quantitative PCR. cDNA was generated using Sepasol RNA I Super $G$ (Nacalai Tesque) according to the manufacturer's instructions. PCR amplification and quantification were performed using SYBR Premix EX TaqII (Takara) and the 7300 Real-Time PCR System (Applied Biosystems). The relative mRNA expression levels were normalized to the Actb expression levels. The nucleotide sequences of the oligos used in the current study are presented in Supplemental Table 1. mRNA from osteoblasts was collected as previously described (48). Femurs were flushed with PBS several times to wash out nonadherent cells. The remaining cells, which mostly consisted of stromal cells and osteoblasts, were lysed by directly injecting Sepasol RNA I Super G into the PBS-flushed femurs.

Luciferase assay. Luciferase assays were performed using the Dual Luciferase Assay System (Promega), and the pRL-SV40 (Promega) plasmid was used as a transfection efficiency control. The 293T cells were transfected with the reporter constructs and the XBP1s and/or NFATc1 expression vectors using FuGene HD (Roche) and incubated for 48 hours. Luciferase activity was measured using GloMax-20/20 (Promega). Transcriptional activity was expressed as the ratio of firefly/Renilla luciferase activity.
ChIP assay. BMMs and RAW264.7 cells were cultured in the presence of sRANKL for 2 days. After incubation, cells were fixed in $1 \%$ PFA/PBS for 10 minutes at room temperature. Chromatin shearing and immunoprecipitation were performed using the ChIP-IT Express Chromatin Immunoprecipitation Kit (Active Motif) according to the manufacturer's instructions. The immunoprecipitated DNA fragments were used as templates for PCR amplification. The nucleotide sequences of the oligos used in the assay are presented in Supplemental Table 2.

The evaluation of cell survival and apoptosis. Cell survival was evaluated using Cell Count Reagent SF (Nacalai Tesque). The rate of apoptotic cells was evaluated by the activity of caspase- 3 and caspase- 7 using the Caspase-Glo 3/7 Assay (Promega).

In vitro osteoclastogenesis assay. $\mathrm{BM}$ cells harvested from the femurs of 9-week-old WT and mutant mice were grown in $\alpha \mathrm{MEM}$ medium supplemented with $10 \%$ fetal calf serum, antibiotics, and 30 $\mathrm{ng} / \mathrm{ml}$ recombinant murine CSF1 (Wako) for 3 days on petri dishes. The adherent cells were used as the BMMs. The BMMs were plated in 48-well plates and further incubated in the presence of sRANKL for 4 days. After incubation, cells were fixed with $10 \%$ formaldehyde/ PBS for 5 minutes, dehydrated with 1:1 ethanol/acetone solution for 1 minute, and air dried for 30 minutes. Cells were stained for TRAP by incubating the fixed cells in acetate buffer ( $0.1 \mathrm{M}$ sodium acetate, pH 5.0) containing naphthol AS-MX phosphate $(0.1 \mathrm{mg} / \mathrm{ml})$, red Fast Red Violet LB Salt (Sigma-Aldrich) $(0.5 \mathrm{mg} / \mathrm{ml})$, and sodium tartrate $(50 \mathrm{mM})$ for 20 minutes as previously described (49). The number 

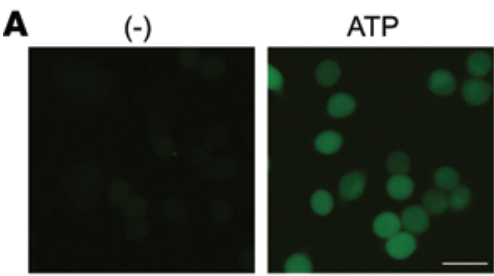

B
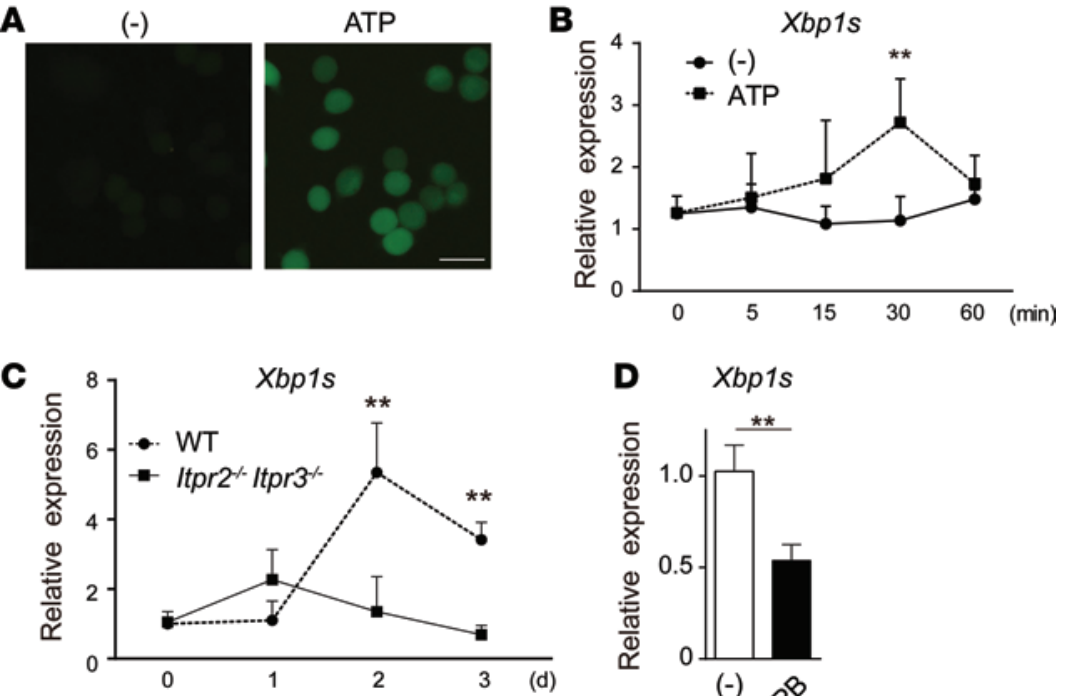

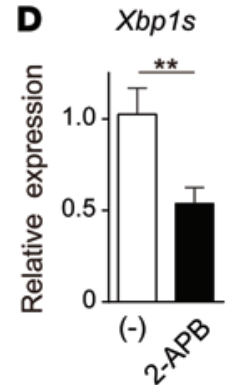

Figure 8. ITPRs-dependent $\mathrm{Ca}^{2+}$ influx activates the IRE1 $\alpha /$ XBP1 pathway during osteoclastogenesis. (A) RAW264.7 cells were incubated with vehicle (-) or ATP $(100 \mu \mathrm{M})$ for 15 minutes. $\mathrm{Ca}^{2+}$ influx into the cytoplasm was visualized using Quest Fluo-8, AM. Scale bar: $20 \mu \mathrm{m}$. (B) Time-course analysis of Xbp1s transcript expression levels in RAW264.7 cells treated with vehicle (-) or ATP $(100 \mu \mathrm{M}) . n=5$ replicates. Values represent mean \pm SD. ${ }^{*} P<0.005$. (C) Relative transcript expression levels of Xbp1s in the WT or Itpr2 ${ }^{-1-}$ Itpr3 ${ }^{-1-}$ BMMs treated with sRANKL for 0 to 3 days. $n=3$ replicates. Values represent mean $\pm \mathrm{SD}$. ${ }^{* *} P<0.005$. (D) BMMs were incubated with sRANKL overnight and subsequently treated with vehicle (-) or 2-aminoethoxydiphenyl borinate (2-APB) $(60 \mu \mathrm{M})$ for 24 hours. The expression levels of Xbp1s transcript were quantitatively analyzed. $n=3$ replicates. Values represent mean $\pm \mathrm{SD} .{ }^{* *} P<0.005$. Statistical analysis was performed using Student's $t$ test. of osteoclasts, which were defined as TRAP-positive multinucleated cells with more than 3 nuclei, was counted under a microscope. Bone resorption activity of osteoclasts was evaluated using Osteo Assay Surface Polystyrene $1 \times 8$ Stripwell Microplate (Corning) per the manufacturer's instructions. Stripwells were scanned using a flatbed scanner, and osteoclast-formed pits were analyzed using ImageJ software (http://imagej.nih.gov/ij/index.html).

Flow cytometry. BM cells collected from WT and $E r n 1^{M x l}$ mice were filtered through a cell strainer $(70 \mu \mathrm{m}$; BD Falcon) to remove debris and were preincubated with an anti-CD16/32 antibody (2.4G2, BD Biosciences) to block nonspecific binding. For detection of the monocyte/ macrophage lineage cells, cells were stained with FITC-conjugated CD11b (M1/70, BioLegend) and allophycocyanin-conjugated Gr-1 (RB6-8C5, BioLegend). For analysis of the osteoclast precursor cells (29), BM mononuclear cells were isolated by centrifugation from total BM cells on Histopaque 1083 (Sigma-Aldrich) following the manufacturer's instructions. BM MNCs were stained with FITC-conjugated CD11b, phycoerythrin-conjugated CD115 (c-Fms, AFS98, BioLegend), and APC-conjugated CD117 (c-Kit, 2B8, BioLegend). Flow cytometric analysis was performed using a Gallios flow cytometer and Kaluza analysis software (Beckman Coulter). Dead cells were excluded from the analyses by propidium iodide staining.

$B M$ transplantation. Donor BM cells were collected from 8-weekold WT or Ern $1^{M x 1}$ mice treated with PIpC at 10 days after birth. The cells were injected into lethally irradiated $(10.5 \mathrm{~Gy})$ recipient WT mice via the retroorbital vein under general anesthesia.

Constructs. The coding region of the Nfatcl gene was obtained by reverse-transcriptase-PCR using mRNA from the BMMs. A Flag-tag sequence was added to the 5 '-terminus of the $\mathrm{Nfatc1}$ gene using a PCRbased method, and the tagged sequence was cloned into the pCMV vector. The expression vector for the HA-tagged XBP1s was generated

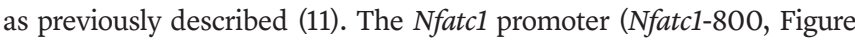
$3 \mathrm{~A}$ ) was cloned by PCR using C57BL/6 genomic DNA as a template and inserted into the pGL3 luciferase reporter vector (Promega). The deletion mutants of the $\mathrm{Nfatc1}$ promoter were generated by a PCR-based method using the KOD-Plus-Mutagenesis Kit (Toyobo) according to the manufacturer's instructions.
Viral gene transfer. The pMXs-IG vector (50) harboring Nfatc1 cDNA and the pLP-VSVG vector were introduced into 293gp packaging cells using FuGENE HD. Viral supernatants were collected on day 4, concentrated using centrifuge, and added to BMMs collected from WT and Ern1 $1^{M x l}$ mice. BMMs were incubated in the presence of CSF1 for 4 days and subsequently incubated in the presence of both sRANKL and CSF1 for 3 to 4 days. At the end of incubation, cells were collected and subjected to analysis. The pMXs-IG vector was generously provided by Toshio Kitamura (The Institute of Medical Science, The University of Tokyo, Tokyo, Japan).

The induction and evaluation of $\mathrm{Ca}^{2+}$ influx. RAW264.7 cells were preincubated with HBSS without FCS for 30 minutes and treated with ATP (Sigma-Aldrich) for 5 to 60 minutes. $\mathrm{Ca}^{2+}$ influx was visualized using Quest Fluo-8, AM (AAT Bioquest) as per the manufacturer's instructions. Total RNA was collected at the designated time points and analyzed for the processing of Xbp1 transcripts.

Immunostaining and image acquisition. BMM cultures were fixed with $4 \%$ PFA/PBS and were immunostained using an anti-XBP1 antibody fol-

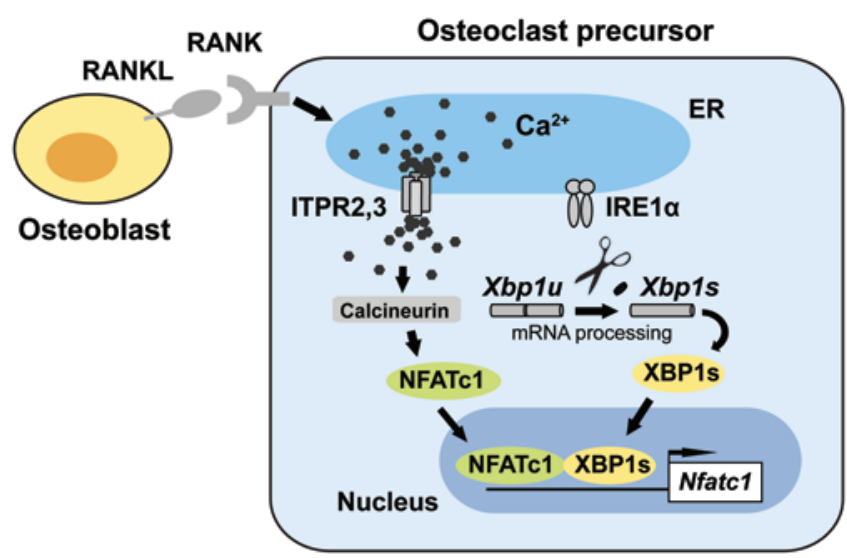

Figure 9. Schematic model proposed by the present study. $\mathrm{Ca}^{2+}$ oscillation is induced by RANKL-RANK signaling and indirectly activates IRE1 $\alpha$, resulting in the splicing of Xbp1u mRNA. Xbp1s gives rise to XBP1s, which functions as a transcription factor for $\mathrm{Nfatc} 1$ and promotes osteoclast differentiation. 
lowed by Alexa Fluor 488-conjugated anti-rabbit IgG (Invitrogen); nuclei were counterstained with DAPI. Images of the cells were acquired with Olympus DP controller software via an Olympus DP70 camera mounted on an Olympus IX71 microscope at room temperature. The objective was a $\times 100 \mathrm{APO} / 1.65$ NA lens. Images were processed with Adobe Photoshop CS6 for contrast correction and to generate merged images.

Statistics. All statistical analyses were performed using Prism 6 (GraphPad Software). Two-tailed Student's $t$ tests for 2 samples assuming equal variances were used to calculate the $P$ values. $P<0.05$ was considered significant.

Study approval. All of the animal experiments in this study were approved by the Institutional Animal Care and Use Committee of the Keio University School of Medicine.

\section{Acknowledgments}

The authors thank Shizue Yamanashi, Kaori Sue, and Mayumi Ito for their technical assistance, Akemi Ito (Ito Bone Science Institute) for advice on bone morphometric analysis, and Taku Saito (The University of Tokyo) for technical help with viral gene transfer. This work was supported, in part, by MEXT KAKENHI (24390358 to K. Horiuchi; 24791566 to T. Tohmonda).

Address correspondence to: Keisuke Horiuchi or Takahide Tohmonda, Department of Orthopedic Surgery, Keio University School of Medicine, Tokyo 160-8582, Japan. Phone 81.3.5363.3812; E-mail: horiuchi@z3.keio.jp (K. Horiuchi), tohmonda@z7.keio.jp (T. Tohmonda).
1. Wang S, Kaufman RJ. The impact of the unfolded protein response on human disease. JCell Biol. 2012;197(7):857-867.

2. Tabas I, Ron D. Integrating the mechanisms of apoptosis induced by endoplasmic reticulum stress. Nat Cell Biol. 2011;13(3):184-190.

3. Hetz C. The unfolded protein response: controlling cell fate decisions under ER stress and beyond. Nat Rev Mol Cell Biol. 2012;13(2):89-102.

4. Ron D, Walter P. Signal integration in the endoplasmic reticulum unfolded protein response. Nat Rev Mol Cell Biol. 2007;8(7):519-529.

5. Hetz C, Martinon F, Rodriguez D, Glimcher LH. The unfolded protein response: integrating stress signals through the stress sensor IRE1 $\alpha$. Physiol Rev. 2011;91(4):1219-1243.

6. Hetz C, Glimcher LH. Fine-tuning of the unfolded protein response: assembling the IRE1 $\alpha$ interactome. Mol Cell. 2009;35(5):551-561.

7. Yoshida H, Matsui T, Yamamoto A, Okada T, Mori K. XBP1 mRNA is induced by ATF6 and spliced by IRE1 in response to ER stress to produce a highly active transcription factor. Cell. 2001;107(7):881-891.

8. Acosta-Alvear D, et al. XBP1 controls diverse cell type- and condition-specific transcriptional regulatory networks. Mol Cell. 2007;27(1):53-66.

9. Lee AH, Iwakoshi NN, Glimcher LH. XBP-1 regulates a subset of endoplasmic reticulum resident chaperone genes in the unfolded protein response. Mol Cell Biol. 2003;23(21):7448-7459.

10. Martinon F, Glimcher LH. Regulation of innate immunity by signaling pathways emerging from the endoplasmic reticulum. Curr Opin Immunol. 2011;23(1):35-40.

11. Tohmonda T, et al. The IRE1 $\alpha$-XBP1 pathway is essential for osteoblast differentiation through promoting transcription of Osterix. EMBO Rep. 2011;12(5):451-457.

12. Sha H, He Y, Yang L, Qi L. Stressed out about obesity: IRE1 $\alpha-\mathrm{XBP} 1$ in metabolic disorders. Trends Endocrinol Metab. 2011;22(9):374-381.

13. Han D, et al. IRE1alpha kinase activation modes control alternate endoribonuclease outputs to determine divergent cell fates. Cell. 2009;138(3):562-575.

14. Niederreiter L, et al. ER stress transcription factor $\mathrm{Xbp} 1$ suppresses intestinal tumorigenesis and directs intestinal stem cells. JExp Med. 2013;210(10):2041-2056.
15. Tohmonda T, et al. The IRE1alpha-XBP1 pathway positively regulates parathyroid hormone (PTH)/ $\mathrm{PTH}$-related peptide receptor expression and is involved in pth-induced osteoclastogenesis. J Biol Chem. 2013;288(3):1691-1695.

16. Boyle WJ, Simonet WS, Lacey DL. Osteoclast differentiation and activation. Nature. 2003;423(6937):337-342.

17. Edwards JR, Mundy GR. Advances in osteoclast biology: old findings and new insights from mouse models. Nat Rev Rheumatol. 2011;7(4):235-243.

18. Teitelbaum SL. Bone resorption by osteoclasts. Science. 2000;289(5484):1504-1508.

19. McInnes IB, Schett G. Cytokines in the pathogenesis of rheumatoid arthritis. Nat Rev Immunol. 2007;7(6):429-442.

20. Steeg PS. Tumor metastasis: mechanistic insights and clinical challenges. Nat Med. 2006;12(8):895-904.

21. Raisz LG. Pathogenesis of osteoporosis: concepts, conflicts, and prospects. JClin Invest. 2005;115(12):3318-3325.

22. Nakashima T, Hayashi M, Takayanagi $H$. New insights into osteoclastogenic signaling mechanisms. Trends Endocrinol Metab. 2012;23(11):582-590.

23. Negishi-Koga T, Takayanagi H. $\mathrm{Ca}^{2+}-\mathrm{NFATc1}$ signaling is an essential axis of osteoclast differentiation. Immunol Rev. 2009;231(1):241-256.

24. Weinstein RS, et al. Promotion of osteoclast survival and antagonism of bisphosphonateinduced osteoclast apoptosis by glucocorticoids. J Clin Invest. 2002;109(8):1041-1048.

25. Patterson RL, Boehning D, Snyder SH. Inositol 1,4,5-trisphosphate receptors as signal integrators. Annu Rev Biochem. 2004;73:437-465.

26. Papandreou I, et al. Identification of an Ire1 $\alpha$ endonuclease specific inhibitor with cytotoxic activity against human multiple myeloma. Blood. 2011;117(4):1311-1314.

27. Iwawaki T, Akai R, Yamanaka S, Kohno K. Function of IRE1 alpha in the placenta is essential for placental development and embryonic viability. Proc Natl Acad Sci U S A. 2009;106(39):16657-16662.

28. Kuhn R, Schwenk F, Aguet M, Rajewsky K. Inducible gene targeting in mice. Science. 1995;269(5229):1427-1429.

29. Arai F, et al. Commitment and differentiation of osteoclast precursor cells by the sequential expression of c-Fms and receptor activator of nuclear factor $\kappa \mathrm{B}$ (RANK) receptors. J Exp Med. 1999;190(12):1741-1754.

30. Willey JS, et al. Risedronate prevents early radiation-induced osteoporosis in mice at multiple skeletal locations. Bone. 2010;46(1):101-111.

31. Kuroda Y, Hisatsune C, Nakamura T, Matsuo K, Mikoshiba K. Osteoblasts induce $\mathrm{Ca}^{2+}$ oscillation-independent NFATc1 activation during osteoclastogenesis. Proc Natl Acad Sci U S A. 2008;105(25):8643-8648.

32. Ruiz A, Matute C, Alberdi E. Endoplasmic reticulum $\mathrm{Ca}(2+)$ release through ryanodine and IP(3) receptors contributes to neuronal excitotoxicity. Cell Calcium. 2009;46(4):273-281.

33. Kiviluoto $S$, et al. Regulation of inositol 1,4,5-trisphosphate receptors during endoplasmic reticulum stress. Biochim Biophys Acta. 2013;1833(7):1612-1624.

34. Lazarowski ER, Boucher RC, Harden TK. Mechanisms of release of nucleotides and integration of their action as P2X-and P2Y-receptor activating molecules. Mol Pharmacol. 2003;64(4):785-795

35. Wagner LE 2nd, Yule DI. Differential regulation of the InsP(3) receptor type- 1 and -2 single channel properties by $\operatorname{InsP}(3), \mathrm{Ca}(2)(+)$ and ATP. JPhysiol. 2012;590(Pt 14):3245-3259.

36. Saito A, et al. Endoplasmic reticulum stress response mediated by the PERK-eIF2(alpha)ATF4 pathway is involved in osteoblast differentiation induced by BMP2. J Biol Chem. 2011;286(6):4809-4818

37. Murakami T, et al. Signalling mediated by the endoplasmic reticulum stress transducer OASIS is involved in bone formation. Nat Cell Biol. 2009;11(10):1205-1211.

38. Saito A, et al. Regulation of endoplasmic reticulum stress response by a BBF2H7-mediated Sec23a pathway is essential for chondrogenesis. Nat Cell Biol. 2009;11(10):1197-1204.

39. Auf $\mathrm{G}$, et al. Inositol-requiring enzyme 1alpha is a key regulator of angiogenesis and invasion in malignant glioma. Proc Natl Acad Sci U S A. 2010;107(35):15553-15558.

40. Fujimoto T, et al. Overexpression of human $\mathrm{X}$-box binding protein 1 (XBP-1) in colorectal adenomas and adenocarcinomas. Anticancer Res. 2007;27(1A):127-131.

41. Chen X, et al. XBP1 promotes triple-negative breast cancer by controlling the HIF1 $\alpha$ pathway. 
Nature. 2014;508(7494):103-107.

42. Carrasco DR, et al. The differentiation and stress response factor XBP-1 drives multiple myeloma pathogenesis. Cancer Cell. 2007;11(4):349-360.

43. Mahoney DJ, et al. Virus-tumor interactome screen reveals ER stress response can reprogram resistant cancers for oncolytic virus-triggered caspase- 2 cell death. Cancer Cell. 2011;20(4):443-456.

44. Mimura N, et al. Blockade of XBP1 splicing by inhibition of IRE1alpha is a promising therapeutic option in multiple myeloma. Blood.
2012;119(24):5772-5781.

45. Vincenz L, Jager R, O’Dwyer M, Samali A. Endoplasmic reticulum stress and the unfolded protein response: targeting the Achilles heel of multiple myeloma. Mol Cancer Ther. 2013;12(6):831-843.

46. Guise TA, Mundy GR. Cancer and bone. Endocr Rev. 1998;19(1):18-54.

47. Futatsugi A, et al. IP3 receptor types 2 and 3 mediate exocrine secretion underlying energy metabolism. Science. 2005;309(5744):2232-2234.
48. Semerad CL, et al. G-CSF potently inhibits osteoblast activity and CXCL12 mRNA expression in the bone marrow. Blood. 2005;106(9):3020-3027.

49. Takahashi N, et al. Osteoclast-like cell formation and its regulation by osteotropic hormones in mouse bone marrow cultures. Endocrinology. 1988;122(4):1373-1382.

50. Kitamura T, et al. Retrovirus-mediated gene transfer and expression cloning: powerful tools in functional genomics. Exp Hematol. 2003;31(11):1007-1014. 